\title{
Implication of the deacetylase sirtuin-1 on synovial angiogenesis and persistence of experimental arthritis
}

Agathe Leblond, ${ }^{1}$ Sonia Pezet, ${ }^{1}$ Anne Cauvet, ${ }^{1}$ Claudine Casas, ${ }_{1}^{1}$ Julie Pires Da Silva, ${ }^{2}$ Roxane Hervé, ${ }^{3,4}$ Gaelle Clavel, ${ }^{3,4,5}$ Sébastien Dumas, ${ }^{6,7}$ Sylvia Cohen-Kaminsky, ${ }^{6,7}$ Natacha Bessis, ${ }^{3,4}$ Luca Semerano, ${ }^{3,4,8}$ Christophe Lemaire, ${ }^{2}$ Yannick Allanore ${ }_{1}^{1,9,10}$ Jérôme Avouac @ 1,9,10

\section{Handling editor Josef $S$ \\ Smolen}

Additional material is published online only. To view, please visit the journal online (http://dx.doi.org/10.1136/ annrheumdis-2020-217377).

For numbered affiliations see end of article.

\section{Correspondence to}

Professor Jérôme Avouac, Rheumatology, Paris Descartes University, Cochin Hospital, Paris 75014, France;

javouac@me.com

Preliminary results from this study have been presented at the American College of Rheumatology congress in 2019: Leblond $A$, et al.

Activation of the desacetylase sirtuin-1 counteracts the activated and proangiogenic profile of endothelial cells in rheumatoid arthritis and alleviates experimental arthritis. Arthritis Rheumatol 2019;71(suppl 10). Abstract no. 53

Received 19 March 2020

Revised 21 April 2020

Accepted 21 April 2020

Published Online First

7 May 2020

\section{ABSTRACT}

Objectives To decipher the phenotype of endothelial cells (ECS) derived from circulating progenitors issued from patients with rheumatoid arthritis (RA)

Methods RA and control ECs were compared according to their proliferative capacities, apoptotic profile, response to tumour necrosis factor (TNF)- $\alpha$ stimulation and angiogenic properties. Microarray experiments were performed to identify gene candidates relevant to pathological angiogenesis. Identified candidates were detected by RT-PCR and western blot analysis in ECs and by immunohistochemistry in the synovium. Their functional relevance was then evaluated in vitro after gene invalidation by small interfering RNA and adenoviral gene overexpression, and in vivo in the mouse model of methyl-bovine serum albumin-(mBSA)-induced arthritis.

Results RA ECs displayed higher proliferation rate, greater sensitisation to TNF- $\alpha$ and enhanced in vitro and in vivo angiogenic capacities. Microarray analyses identified the NAD-dependent protein deacetylase sirtuin-1 (SIRT1) as a relevant gene candidate. Decreased SIRT1 expression was detected in RA ECs and synovial vessels. Deficient endothelial SIRT1 expression promoted a proliferative, proapoptotic and activated state of ECs through the acetylation of p53 and p65, and lead the development of proangiogenic capacities through the upregulation of the matricellular protein cysteinerich angiogenic protein-61. Conditional deletion of SIRT1 in ECs delayed the resolution of experimental methyl-bovine serum albumin-(mBSA)-induced arthritis. Conversely, SIRT1 activation reversed the pathological phenotype of RA ECs and alleviates signs of experimental mBSA-induced arthritis.

Conclusions These results support a role of SIRT1 in RA and may have therapeutic implications, since targeting angiogenesis, and especially SIRT1, might be used as a complementary therapeutic approach in RA.

\section{INTRODUCTION}

Rheumatoid arthritis (RA) is the most common chronic inflammatory arthritis. ${ }^{1}$ The synovium is the primary site of the inflammatory process, which, if untreated, leads to irreversible damages to the adjacent cartilage and bone. One of the most noticeable features of rheumatoid synovitis is the amount of synovial vascularisation, which is critical for synovial proliferation and invasiveness. Increased vascular density in RA results from the pathological

\section{Key messages}

What is already known about this subject?

- Angiogenesis through the activation of endothelial cells (ECs) is a crucial event to promote the development of the pathological synovium in rheumatoid arthritis (RA).

What does this study add?

- This work provides the first experimental evidence of a proliferative, activated and proangiogenic profile of RA ECs.

- The deacetylase sirtuin-1 (SIRT1) was identified as a relevant actor involved in all the main pathological features of those cells, and SIRT1 expression is markedly reduced in ECs and synovial vessels of patients with RA.

- Endothelial SIRT1 invalidation reproduces the phenotype of RA ECs and exacerbates experimental arthritis, and these effects were reversed by SIRT1 overexpression.

\section{How might this impact on clinical practice or} future developments?

- These results may have direct therapeutic implications, since targeting angiogenesis, and especially SIRT1, might be used as an adjuvant treatment of RA.

activation of angiogenesis and vasculogenesis by secreted mediators of tissue infiltrating inflammatory cells that lead to the unrestrained formation of new blood vessels. ${ }^{2-5}$ However, recent evidence suggests a primary involvement of angiogenesis in the initiation of tissue inflammation, prior to infiltration of inflammatory cells. ${ }^{6}$ These results add further data to the accumulating evidence on the relevance of endothelial cells (ECs) in the pathophysiology of inflammation.

Formation of new blood vessels consists of several complementary processes including activation, proliferation and migration of ECs. Our group has developed a non-invasive innovative method to obtain culture ECs derived from circulating progenitors, which represent valuable tools to study endothelial biology. ${ }^{7-10}$

To gain insights into the implication of angiogenesis and vasculogenesis in RA, our aims were to i) study the properties of circulating 
progenitor-derived ECs issued from patients with RA, ii) decipher gene expression profiles of those cells to identify new potentially relevant angiogenic candidates and iii) study the consequences of angiogenic candidate invalidation/overexpression on EC functional properties and on experimental arthritis.

\section{PATIENTS AND METHODS}

An extended 'Patients and methods' section is available in the online supplementary data.

\section{Patient samples and synovial tissue}

This study involved 29 patients with RA fulfilling the 1987 American College of Rheumatology (ACR) or the 2010 ACR/European League Against Rheumatism classification for RA ${ }^{11} 12$ and 18 age-matched and gender-matched controls (online supplementary tables S1 and S2).

\section{Microarray analysis}

Microarray analysis was performed on 18 patients with RA and 11 controls. Affymetrix Microarray technology was used to analyse gene expression levels (Affymetrix GeneChip Human
Exon 1.0 ST Arrays). Labelling and microarray processing were performed according to the manufacturer's protocol. ${ }^{89}$

RNA interference assay and adenovirus transduction

ECs were seeded and transfected with deacetylase sirtuin-1 (SIRT1) small interfering (siRNA) (20 nM; Qiagen, Hilden, Germany) or control siRNA (20 nM). Adenovirus amplification (gift from Dr Christophe Lemaire) was performed using the Vivapure AdenoPACK 20 kit (Progen, Heidelberg, Germany).

SIRT1 activity assay

The activity of SIRT1 from RA and control ECs was tested with a Biomol SIRT1 fluorescence assay kit (AK-555; Biomol, Farmingdale, New York, USA).

Quantitative RT-PCR, western blot analysis, ELISA, immunohistochemistry, immunofluorescence, flow cytometry These methods were performed with reagents and standard techniques described in online supplementary data (online supplementary tables S2 and S3).
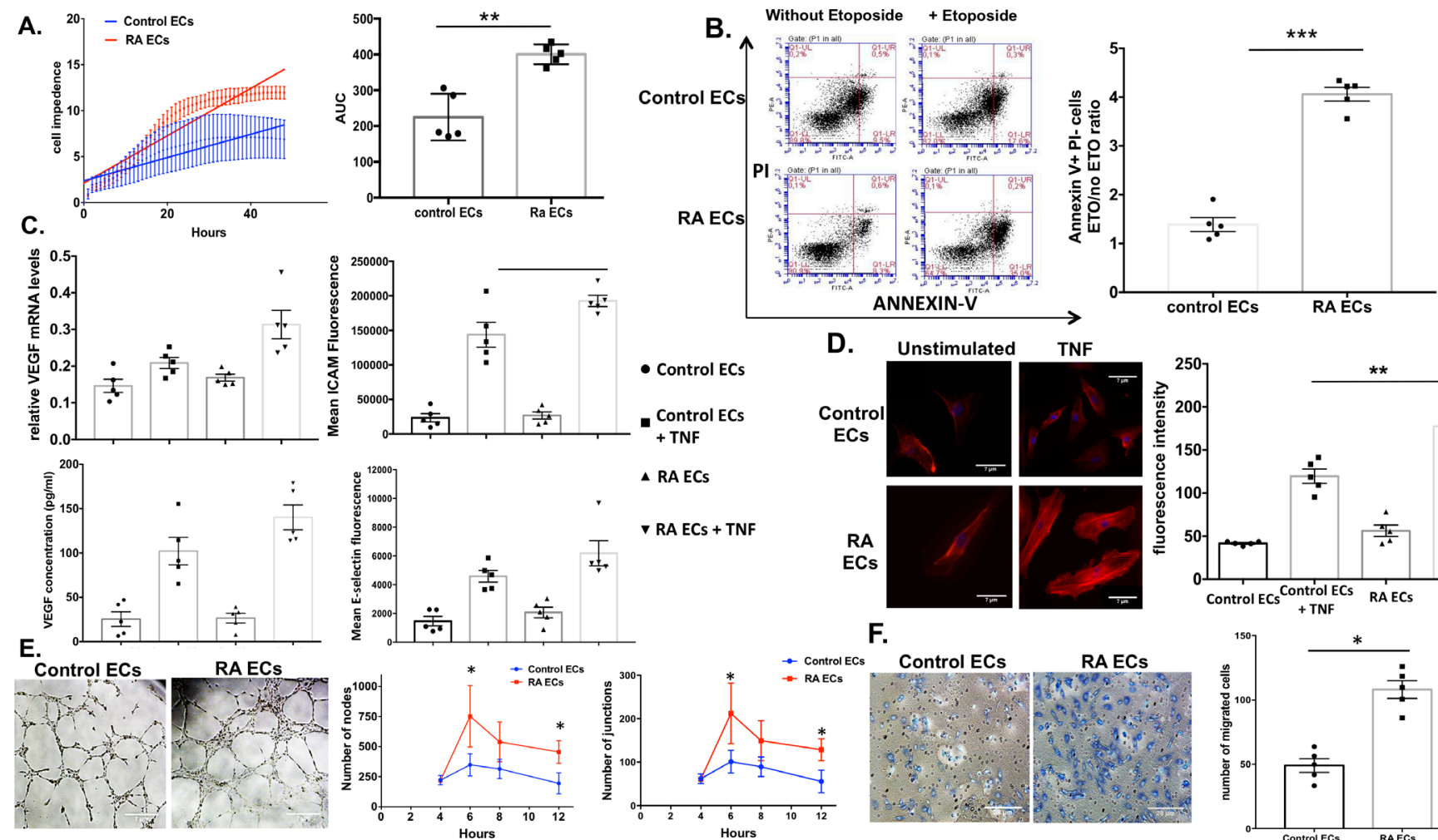

RAECS

RA ECs + TNF
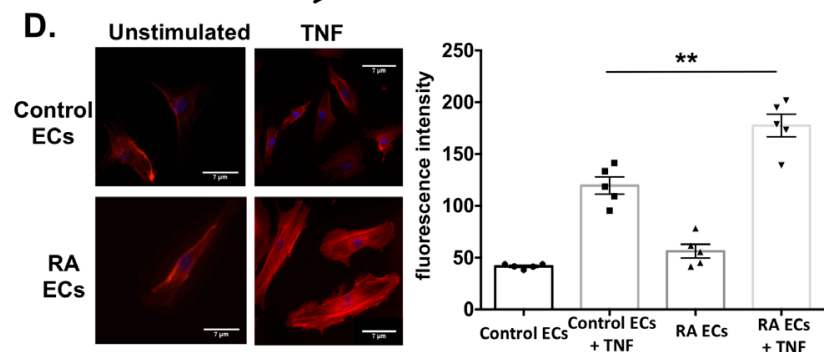

F.
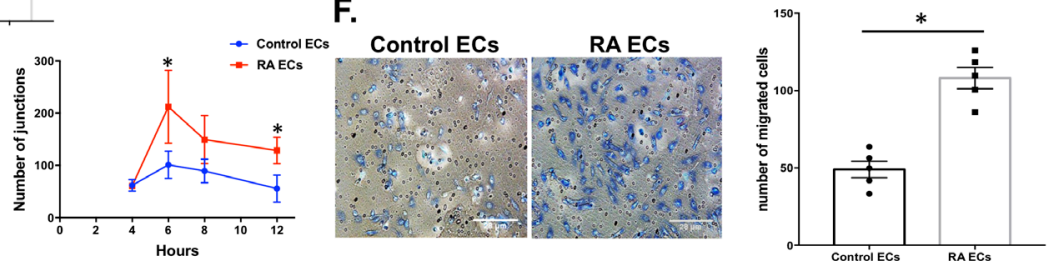

Figure 1 Functional properties of rheumatoid arthritis (RA) and control endothelial cells (ECS). (A) Cell impedance measured by xCELLigence system in RA and control ECs. Y-axis shows the cell impedance and the area under the curve (AUC) of cell impedance in RA and control ECs. (B) Representative flow cytometry dot plots with double Annexin V-FITC/PI staining for RA and control ECs following etoposide-induced apoptosis $(100 \mu \mathrm{M}$ for 24 hours). Y-axis represents the $\mathrm{x}$-fold change of Annexin V-FITC+/PI- cells after etoposide exposure (100 $\mu \mathrm{M}$ for 24 hours) in RA and control ECs. (C.) Relative vascular endothelial growth factor (VEGF) mRNA levels (qRT-PCR), VEGF concentration in culture cell supernatants (ELISA) and intercellular adhesion molecule (ICAM)-1/E-selectin expression (flow cytometry) in RA and control ECs following tumour necrosis factor (TNF)- $\alpha$ exposition ( $50 \mathrm{ng} / \mathrm{mL}$ for 5 hours). (D) Representative images of stress fibre formation on TNF- $\alpha$ stimulation $(50 \mathrm{ng} / \mathrm{mL}$ for 5 hours) (scale bar $=7 \mu \mathrm{m})$. Nuclei are stained with DAPI (blue). Y-axis shows fluorescence intensity quantified by ImageJ. (E) Representative images of tube formation at 6 hours in RA and control ECs (scale bar $=7 \mu \mathrm{m}$ ). Y-axis shows the node and junction numbers at 4, 6, 8 and 12 hours. (F) Representative images of cell migration in modified Boyden chamber following VEGF activation $(50 \mathrm{ng} / \mathrm{mL}$ for 6 hours) in RA and control ECs (scale bar=28 $\mu \mathrm{m}) ; \mathrm{Y}$-axis shows the number of migrated cells. ECs from five independent patients with RA and five independent controls were used in all experiments. All data are shown as the mean \pm SEM. ${ }^{*} \mathrm{P}<0.05,{ }^{* *} \mathrm{p}<0.01,{ }^{* *} \mathrm{p}<0.001$ determined by Student's t-test $(\mathrm{A}, \mathrm{B}, \mathrm{E}, \mathrm{F})$ or one-way analysis of variance with Tukey's post hoc test $(C, D)$ for experiments including more than two groups in one experiment. Data are representative of two independent experiments. 
A.

C.

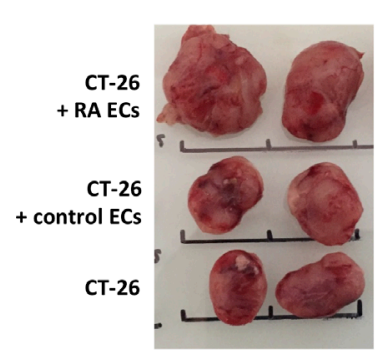

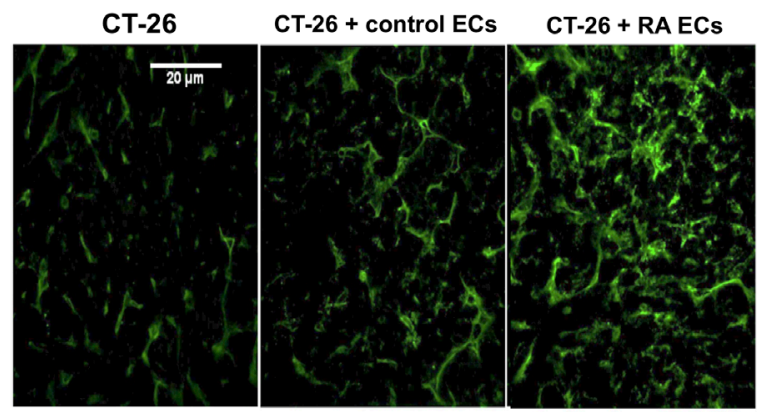

D.

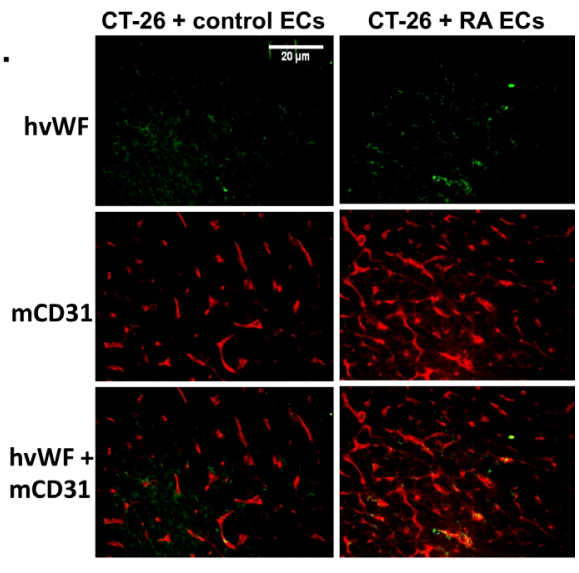

F.

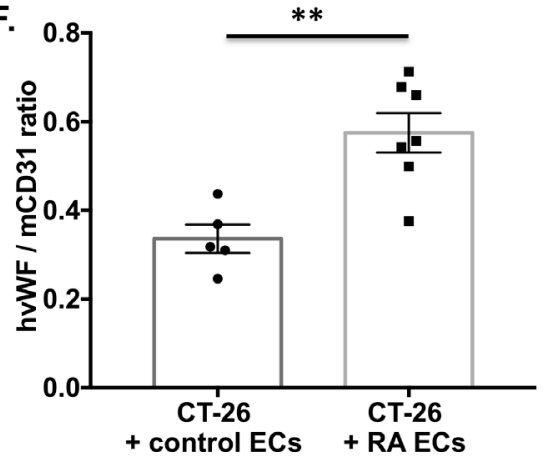

Figure 2 Size and neovessel density in mice that received transplants of CT-26 cells alone or in combination with control or rheumatoid arthritis (RA) endothelial cells (ECs). (A, B) Representative subcutaneous tumours from mice that received transplants and the volume of tumours that developed. Each data point represents a single mouse (receiving a transplant from a single patient). (C) Representative images of intratumoural vessel density assessed by immunofluorescence for murine CD31 (green) (scale bar=20 $\mu \mathrm{m}$ ). (D) Quantification of murine CD31 fluorescence intensity. (E) Representative images of the incorporation of human ECs in mouse vascular structures, assessed by double labelling for human von Willebrand factor (green) and murine CD31 (red) (scale bar=20 $\mu \mathrm{m}$ ). (F) Quantification of the number of human von Willebrand factor positive cells reported to the number of murine CD31-positive cells. A total of 17 mice were used: 7 injected with CT26 cells and RA ECs, 5 with CT26 cells and control ECs and 5 with CT-26 cells alone. All data are shown as the mean \pm SEM of a single experiment. ${ }^{*} \mathrm{P}<0.05,{ }^{*} \mathrm{p}<0.01$ determined by Student's $\mathrm{t}$-test (F) or oneway analysis of variance with Tukey's post hoc test $(B, D)$ for experiments including more than two groups in one experiment.

\section{xCELLigence system}

Cell proliferation was monitored using the xCELLigence RTCA MP (ACEA Biosciences, San Diego, California, USA), which measures cell impedance in real and continuous time.

\section{Angiogenic assays}

These assays consisted of tube formation in matrigel matrix and migration in modified Boyden chambers. ${ }^{7}$

\section{Generation of conditional endothelial SIRT1 KO mice}

To generate C57BL/6 mice carrying both the TEK-Cre-ER ${ }^{\mathrm{T} 2}$ and the SIRT1 $1^{\text {flox } \Delta \mathrm{E} 4 / \text { flox } \Delta \mathrm{E} 4}$ alleles, SIRT1 $1^{\text {flox } \Delta \mathrm{E} 4 / \text { flox } \Delta \mathrm{E} 4}$ mice were crossed with TEK-Cre-ER ${ }^{\mathrm{T} 2}$ mice. After two generations, homozygote SIRT1 Flox/Flox mice expressing the Cre recombinase were obtained. Excision of SIRT1 exon 4 was induced by tamoxifen diet $(400 \mathrm{mg} / \mathrm{kg}$ ) (Envigo, Gannat, France) (online supplementary figure S1).

\section{Mouse model of tumour neovascularisation}

Syngeneic murine colon carcinoma CT-26 cells $\left(2.5 \times 10^{5}\right.$ cells $)$ (LGC standards, Molsheim, France) were transplanted subcutaneously into the backs of mice with severe combined immunodeficiency, alone $(n=5)$ or in combination with control ECs $\left(5 \times 10^{3}\right.$ cells) $(n=7)$ or RA ECs $\left(5 \times 10^{3}\right.$ cells) $(n=7)$. The subcutaneous tumours were removed 15 days after tumour transplantation.
Mouse model of antigen-induced arthritis

This model was induced on a total of 32 mice: 6 SIRT1 Flox/Flox; WT/WT mice, 7 SIRT1 Flox/Flox; Cre/WT mice and 19 C57BL/6 mice. An active group of $9 \mathrm{C} 57 \mathrm{BL} / 6$ mice received daily intraperitoneal injections of resveratrol (Sigma-Aldrich) $(20 \mathrm{mg} / \mathrm{kg} / \mathrm{day}$ in $100 \mu \mathrm{L}$ of phosphate-buffered saline (PBS)) and a second control group of $10 \mathrm{C} 57 \mathrm{BL} / 6$ mice received daily intraperitoneal injections of $100 \mu \mathrm{L}$ of PBS, starting the day of first injection of mBSA until mouse sacrifice.

\section{Statistics}

All analysis was performed with GraphPad Prism 7.0 (GraphPad, San Diego, California, USA). All data are expressed as mean values \pm SEM. Multiple group comparisons were analysed by oneway analysis of variance with Tukey's post hoc test. Unpaired or paired t-test was used for a two-group comparison. $\mathrm{P}<0.05$ (all two-sided) was considered to be statistically significant.

\section{RESULTS}

\section{ECs issued from patients with RA display an activated and} proangiogenic profile

RA ECs displayed higher proliferation rate compared with control ECs (figure 1A), with a significantly different slope of the curves for each dataset (best-fit values: $0.26 \pm 0.01$ vs $0.19 \pm 0.01$, $\mathrm{p}<0.001$ ) and area under the proliferation curve (figure $1 \mathrm{~A}) . \mathrm{RA}$ 
A.

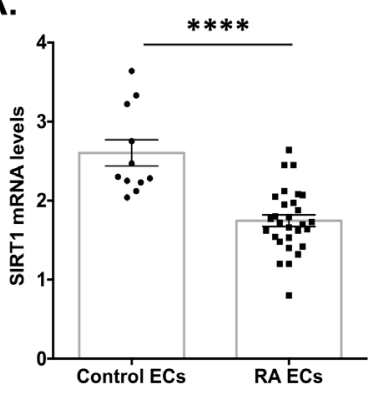

G.

Control

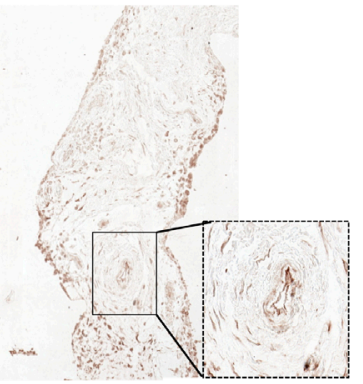

B.

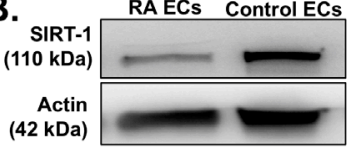

C.

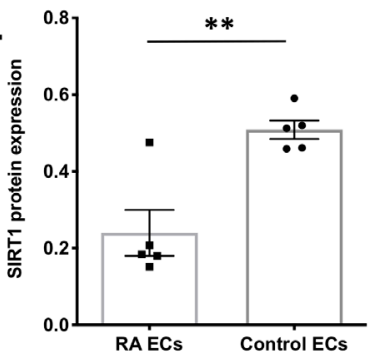

D.
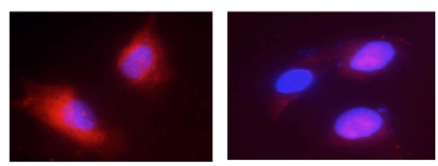

E.

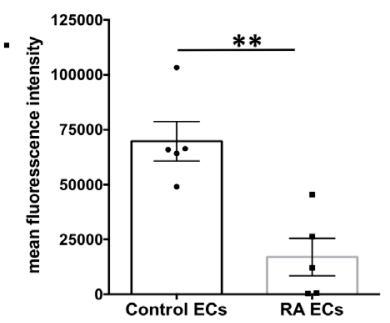

F.

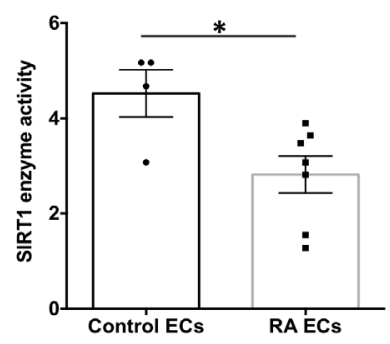

H.

RA

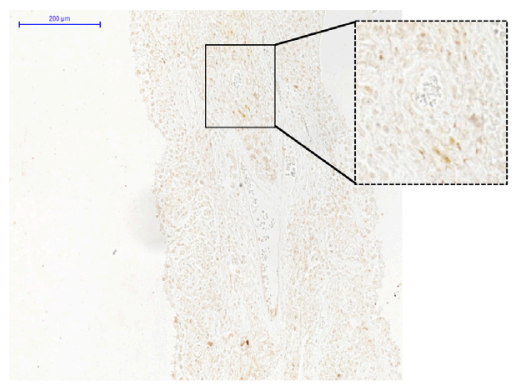

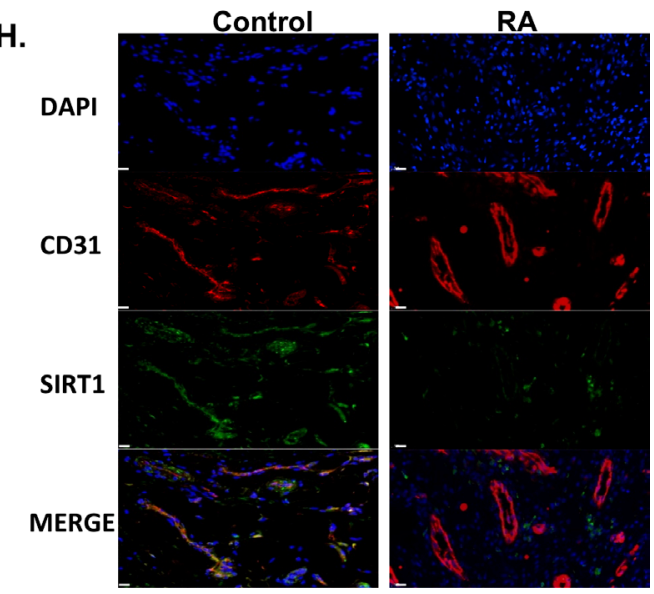

Figure 3 Cellular and tissular sirtuin-1 (SIRT1) expression. (A) SIRT1 mRNA levels quantified by qRT-PCR in rheumatoid arthritis (RA) ( $\mathrm{n}=29)$ and control ( $n=11$ ) endothelial cells (ECs). (B) Cell extracts from cultured RA and control ECs were immunoblotted for SIRT1. (C) Quantification of antiSIRT1 by western blot analysis. (D) Representative immunofluorescence staining for SIRT1 in RA and control ECs (scale bar=10 $\mu \mathrm{m})$. Nuclei are stained with DAPI (blue). (E) Quantification of fluorescence intensity with ImageJ. (F) Quantification of SIRT1 activity in RA ( $n=7)$ and control ( $n=4)$ ECs. (G) Representative immunohistochemistry staining for SIRT1 in lesional synovial tissue issued from a patient with RA and a control (scale bar=200 $\mu \mathrm{m}$ ). (H) Representative double labelling by immunofluorescence for SIRT1 (red) and CD31 in the synovial tissue taken from a patient with RA and a control (green). Nuclei are stained with DAPI (blue) (scale bar $=20 \mu \mathrm{m}$ ). ECs from five independent patients with RA and five independent controls were used in all experiments, unless stated otherwise. Synovial tissue from five independent patients with RA and three independent controls was used. All data are shown as the mean \pm SEM of one experiment. ${ }^{*} P<0.05,{ }^{* *} p<0.01,{ }^{* * *} p<0.0001$ determined by Student's t-test. Data are representative of two independent experiments.

ECs exhibited a pro-apoptotic profile: the number of Aannexin $\mathrm{V}+/ \mathrm{PI}-$ cells was increased by 2.92 -fold in RA compared with controls on exposure to etoposide $(\mathrm{p}<0.001)$ (figure $1 \mathrm{~B})$. RA ECs presented increased sensitisation to tumour necrosis factor (TNF)- $\alpha$. On stimulation with rhTNF- $\alpha$, vascular endothelial growth factor (VEGF) mRNA levels and concentrations released in cell culture supernatants increased in RA ECs compared with control ECs (1.50-fold, $\mathrm{p}=0.03$ and 1.28 -fold, $\mathrm{p}=0.160$, respectively) (figure $1 \mathrm{C}$ ). The expression of adhesion molecules and the formation of stress fibres on TNF- $\alpha$ stimulation were also strikingly more prominent in RA ECs (figure 1D). RA ECs also displayed greater angiogenic properties in vitro, with accelerated tube formation (figure 1E) and increased migration capacities (figure $1 \mathrm{~F}$ ). We next proceeded with the evaluation of proangiogenic capacities of RA ECs in experimental neoangiogenesis using the mouse model of tumour neovascularisation. When CT-26 cells were used transplanted with RA ECs, tumour growth was markedly stronger versus when they were transplanted with control ECs (mean $\pm S D, 3.02 \pm 0.92 \mathrm{~cm}^{3}$ vs $1.83 \pm 0.36 \mathrm{~cm}^{3}$; $\mathrm{p}=0.005$ ) (figure $2 \mathrm{~A}-\mathrm{B}$ ). Neovessel density was significantly increased in tumours that developed when CT-26 cells were transplanted with RA ECs, as compared with those transplanted with control ECs (figure 2C-D), supporting the greater in vivo capacity of these cells to promote neovascularisation. We observed a correlation between neovessel density and tumour size $(r=0.87, p<0.001)$, suggesting that neovessel formation is a potent contributor to tumour growth. In addition, a significantly higher proportion of transplanted human RA ECs expressing the human-specific mature-EC marker von Willebrand factor had been incorporated into the endothelium compared with control ECs (figure 2E-F).

\section{Decreased cellular and tissular expression and activity of the NAD-dependent deacetylase sirtuin-1 in RA}

We next compared gene expression profiles of unstimulated RA and control ECs. Unsupervised analyses by hierarchical clustering allowed a correct segregation between patients with RA and controls (online supplementary figure S2A). Volcano plot illustrated fold-differences in individual gene expression and associated $\mathrm{p}$ values (negative $\log 10$ ) (online supplementary figure S2B). Supervised analyses identified 879 differentially expressed genes, with a significant enrichment in functional groups related to cell cycle (94 genes), cell death and survival (205 genes), cellular growth and proliferation (143 genes) and cell morphology (143 genes). A list of top genes and their upstream regulators, chosen according resulting $\mathrm{p}$ values $(<0.05)$, fold change and biological relevance (online supplementary table S4), were then entered 
A.

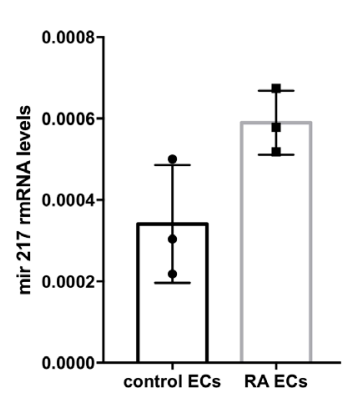

B.

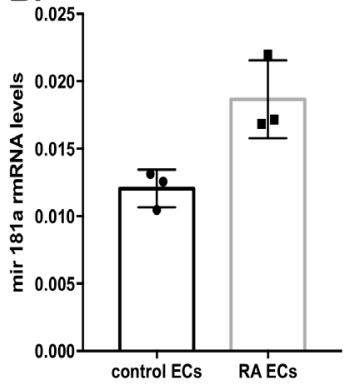

C

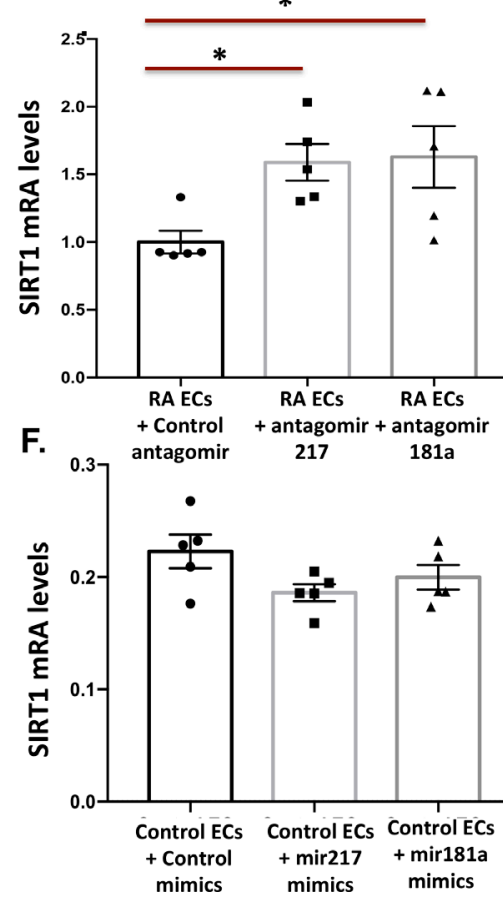

D.

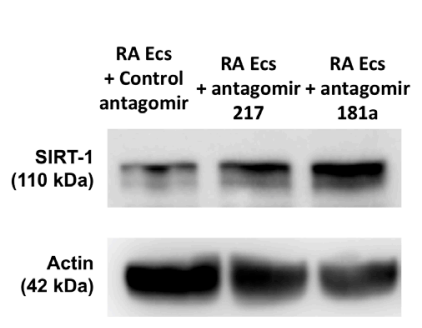

G.

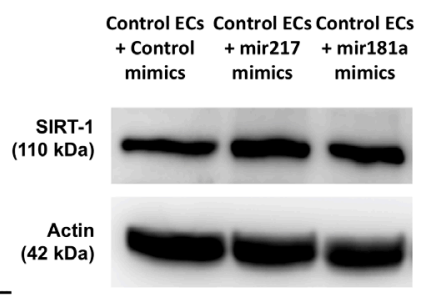

E.

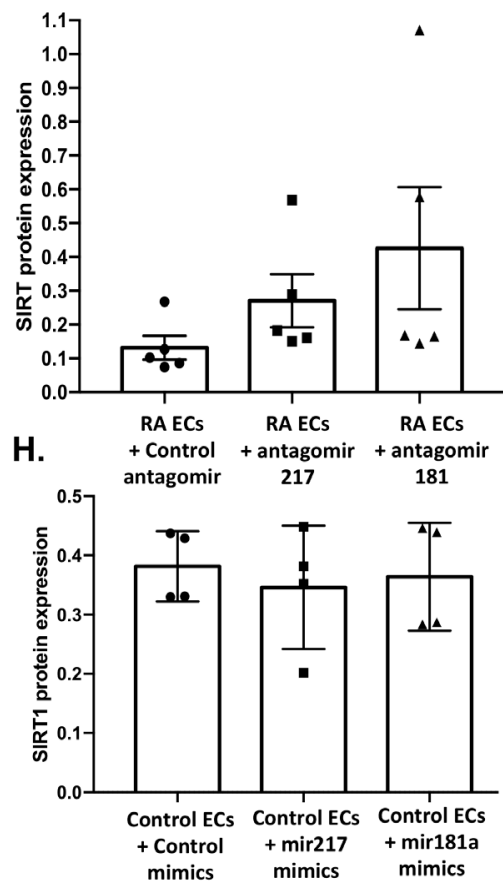

Figure 4 Effects of miR-217 and miR-181 a modulation on sirtuin-1 (SIRT1) expression in rheumatoid arthritis (RA) and control endothelial cells (ECS). (A, B) mRNA levels of miR-217 (A) and miR-181a (B) quantified by qRT-PCR in RA and control ECs ( $n=3$ each). (C) SIRT1 mRNA levels quantified by qRT-PCR in RA ECs transfected with control antagomiR, antagomiR-217 or antagomiR-181a ( $n=5$ each). (D) Cell extracts from cultured RA ECs transfected with control antagomiR, antagomiR-217 or antagomiR-181a ( $n=5$ each) were immunoblotted for SIRT1. (E) Quantification of anti-SIRT1 by western blot analysis. (F) SIRT1 mRNA levels quantified by qRT-PCR in control ECs transfected with control mimics, miR-217 mimics or miR-181a mimics ( $n=5$ each). (G) Cell extracts from cultured control ECs transfected with control mimics, miR-217 mimics or miR-181a mimics ( $n=5$ each) were immunoblotted for SIRT1. (H) Quantification of anti-SIRT1 by western blot analysis. ${ }^{*} \mathrm{P}<0.05$ determined by one-way analysis of variance with Tukey's post hoc test. Data are representative of two independent experiments.

into the biological database STRING to construct a functional protein association network. This analysis revealed an interaction network centred on the NAD-dependent SIRT1 (online supplementary figure S2C), implicated in cell proliferation and survival, inflammation and angiogenesis. SIRT1 mRNA and protein levels were decreased by $33 \%(p<0.001)$ and $53 \%(p=0.003)$, respectively (figure $3 \mathrm{~A}-\mathrm{C}$ ). Immunocytofluorescence showed that SIRT1 expression was localised to the cytoplasm (figure 3D-E). Together with reduced expression, SIRT1 deacetylase activity was significantly decreased by $38 \%$ in RA ECs $(p=0.039)$ (figure 3F) and the acetylation of the SIRT1-regulated transcription factors p 53 and p 65 was increased in RA ECs (online supplementary figure S3A-D). Epigenetic modifications by microRNAs are an important mechanism of SIRT1 expression and activity regulation. ${ }^{13-15}$ The expression of miR-217 and miR-181a were increased in RA ECs (figure 4A-B). Moreover, SIRT1 expression was restored in RA ECs on transfection with antagomiR-217 and 181a (figure 4C-E). Conversely, SIRT1 levels did not markedly diminish in control ECs transfected with miR-217 or miR-181a mimics (figure 4F-H). Finally, SIRT1 expression was reduced in the synovial tissue of patients with RA (figure 3G). Double labelling with SIRT1 and CD31 revealed a markedly reduced SIRT1 expression in synovial vessels (figure $3 \mathrm{H}$ ).

\section{SIRT1 silencing enhances control EC turnover, activation and proangiogenic properties}

To assess whether decreased SIRT1 expression may contribute to the pathological profile of ECs, we transfected control ECs with SIRT1 siRNA (online supplementary figure S4A-B).

\section{SIRT1 silencing promotes control EC proliferation and mediates apoptosis}

The proliferation rate of SIRT1 siRNA-transfected control ECs was significantly higher than mock-transfected cells (slope analysis with best-fit values: $0.14 \pm 0.01$ vs $0.12 \pm 0.01, \mathrm{p}=0.044$ ) (figure 5A). Together with increased cell proliferation, SIRT1 invalidation was also associated with increased EC apoptosis. On etoposide exposure, the number of apoptotic Annexin V+/ PI- cells increased by 1.84 -fold in SIRT1 siRNA-transfected cells $(\mathrm{p}=0.038)$ (figure 5B).

\section{SIRT1 silencing leads to increased sensitisation of control ECs to TNF- $\alpha$ \\ On TNF- $\alpha$ stimulation, VEGF mRNA levels increased by 1.7 -fold $(p=0.009)$ in control ECs, and the release of VEGF in culture cell supernatants increased by 3.4-fold $(\mathrm{p}=0.021)$ (figure 5C). Transfection with SIRT1 siRNA strikingly enhanced the effects of TNF- $\alpha$ on VEGF synthesis at the mRNA and protein levels (figure 5C). Consistent with this finding, SIRT1 knockdown conducted to a more pronounced TNF- $\alpha$-dependent expression of the adhesion molecules intercellular adhesion molecule- 1 and E-selectin (figure 5C) and to the stimulation of actin stress fibre formation (figure 5D).}

SIRT1 silencing amplifies control EC proangiogenic properties Transfection of control ECs with SIRT1 siRNA led to accelerated tube formation (figure $5 \mathrm{E}$ ) and greater migration capacities on VEGF stimulation (figure 5F). 

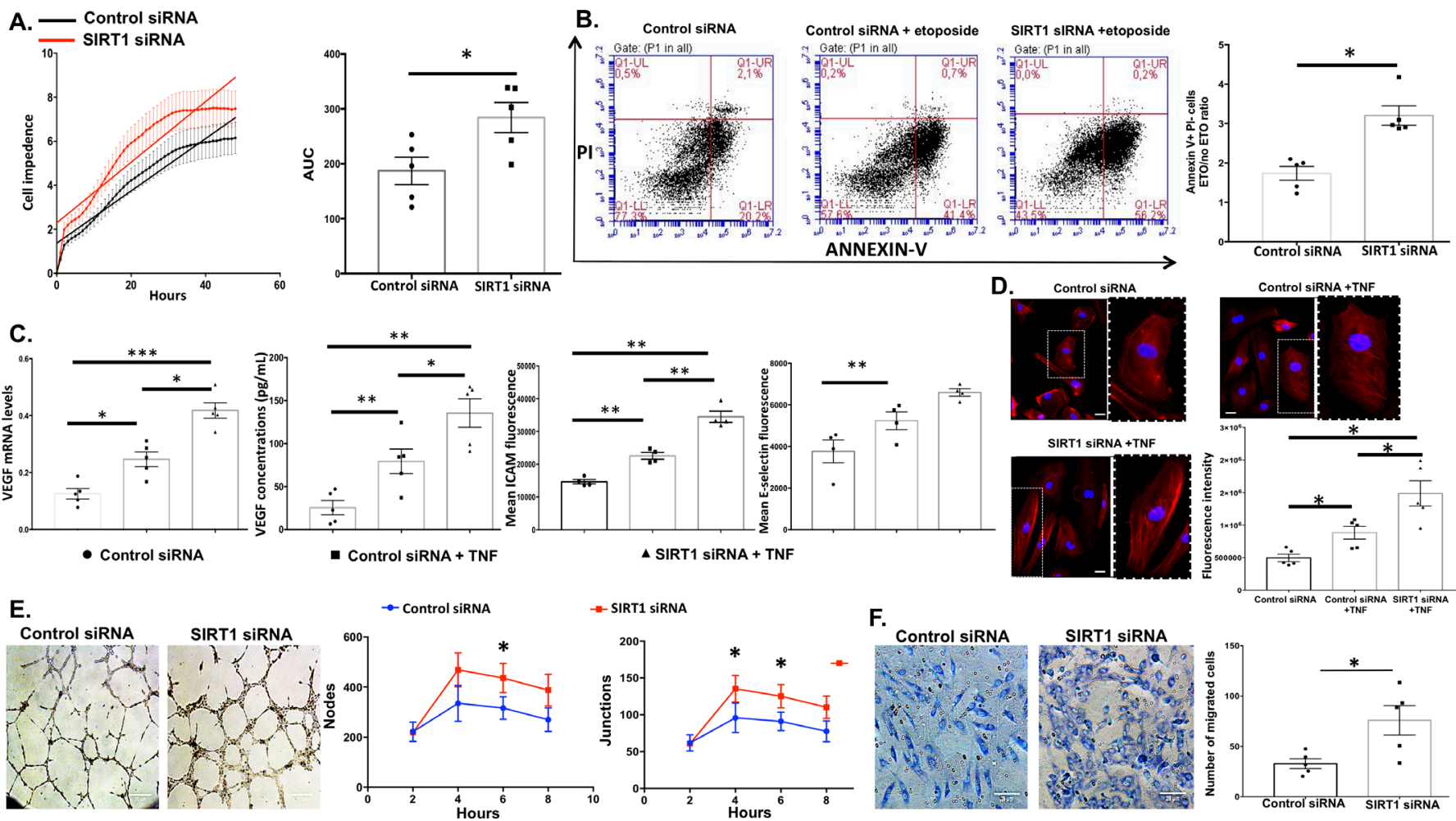

E.

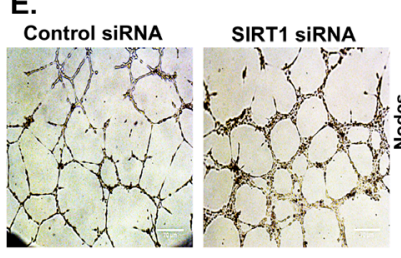

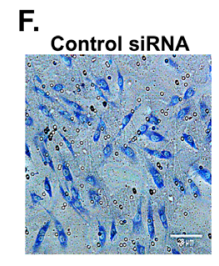

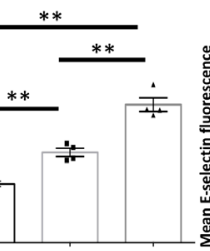

$\triangle$ SIRT1 SIRNA + TNF
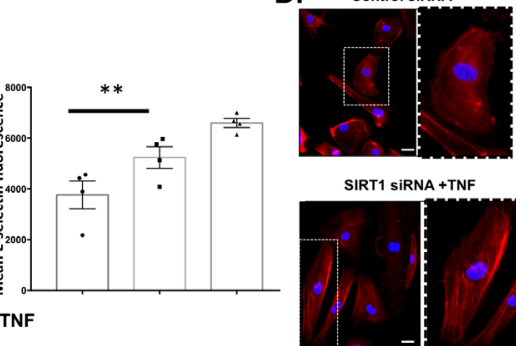

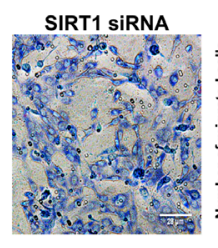

F.

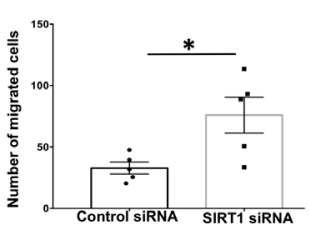

Figure 5 Effects of sirtuin-1 (SIRT1) inhibition on cell proliferation, survival, activation and angiogenic properties in control endothelial cells (ECs) transfected with SIRT1 small interfering (siRNA). (A) Cell impedance measured by xCELLigence system. Y-axis shows the area under the curve of cell impedance in mock-transfected and SIRT1-transfected control ECs. (B) Representative flow cytometry dot plots with double Annexin V-FITC/ PI staining for mock-transfected and SIRT1-transfected control ECs following etoposide-induced apoptosis (100 $\mu \mathrm{M}$ for 24 hours). Y-axis represents the $x$-fold change of Annexin V-FITC+/PI- cells after etoposide exposure (100 $\mu \mathrm{M}$ for 24 hours) in mock-transfected and SIRT1-transfected control ECs. (C) Relative vascular endothelial growth factor (VEGF) mRNA levels (qRT-PCR), VEGF concentration in culture cell supernatants (ELISA) in mocktransfected and SIRT1-transfected control ECs following tumour necrosis factor (TNF)- $\alpha$ exposition (50 ng/mL for 5 hours). Intercellular adhesion molecule (ICAM)-1 and E-selectin expression assessed by flow cytometry in mock-transfected $(n=4)$ and SIRT1-transfected $(n=4)$ control ECs following TNF- $\alpha$ exposition ( $50 \mathrm{ng} / \mathrm{mL}$ for 5 hours). (D) Representative images of stress fibre formation on TNF- $\alpha$ stimulation $(50 \mathrm{ng} / \mathrm{mL}$ for 5 hours) (scale $\mathrm{bar}=20 \mu \mathrm{m}$ ). Nuclei are stained with DAPI (blue). Y-axis shows the fluorescence intensity quantified by ImageJ. (E) Representative images of tube formation at 6 hours in mock-transfected and SIRT1-transfected control ECs (scale bar=70 $\mu \mathrm{m}$ ). Y-axis shows the node and junction numbers at 2, 4, 6 and 8 hours. (F) Representative images of cell migration in modified Boyden chamber following VEGF activation ( $50 \mathrm{ng} / \mathrm{mL}$ for 6 hours) in mocktransfected and SIRT1-transfected control ECs (scale bar $=28 \mu \mathrm{m})$; Y-axis shows the number of migrated cells. ECs from five independent patients with RA and five independent controls were used in all experiments, unless stated otherwise. All data are shown as the mean $\pm \mathrm{SEM}$. ${ }^{*} \mathrm{P}<0.05,{ }^{* *} \mathrm{p}<0.01$, ${ }^{* * *} p<0.001$ determined by Student's $t$-test $(A, B, E, F)$ or one-way analysis of variance with Tukey's post hoc test $(C, D)$ for experiments including more than two groups in one experiment. Data are representative of two independent experiments.

\section{Mechanism of action of SIRT1 in ECs}

Given that $\mathrm{p} 53$ is required for etoposide-induced apoptosis in different cell types, ${ }^{16}$ we aimed to determine whether the effects of SIRT1 knockdown on EC apoptosis were mediated by increased p53 acetylation. As expected, etoposide exposure led to increased acetylated (Ac)-p53/total p53 ratio in mocktransfected ECs. The transfection of control ECs with SIRT1 siRNA significantly enhanced p53 acetylation (figure 6A).

Since SIRT1 physically interacts with the p65 subunit of nuclear factor kappa B (NF- $\mathrm{KB}$ ) and inhibits transcription by deacetylating RelA/p65 at lysine $310,{ }^{17}$ we aimed to determine whether the greater sensitisation of ECs invalidated for SIRT1 to TNF- $\alpha$ might be related to increased p 65 acetylation. As expected, treatment with TNF- $\alpha$ stimulated the expression of total p65 in EC nuclear extracts (figure 6B). Transfection of TNF- $\alpha$-stimulated control ECs with SIRT1 siRNA did not modify the expression of total p65, but led to a significant 4.9-fold increase in the Ac-p65/ total p65 ratio (figure 6B).

The matricellular protein cysteine-rich angiogenic protein 61 (CYR61) is a strong regulator of angiogenesis, whose expression is regulated by SIRT1 in synovial and dermal fibroblasts. ${ }^{18-20}$ In RA ECs, reduced expression of SIRT1 was associated with increased CYR61 expression. Indeed, The mRNA and protein expression of CYR61 were markedly increased in RA ECs (figure 6C-E) and CYR61 concentrations measured in EC culture supernatants were significantly higher in patients with RA compared with controls (figure 6F). Moreover, the transfection of control ECs with SIRT1 siRNA was associated with a significant increase of CYR61 protein (figure 6G).

\section{Upregulation of SIRT1 reverses the proliferative, activated and proangiogenic profile of RA ECs}

We next aimed to evaluate whether the restoration SIRT1 expression and enzyme activity would reverse the pathological profile of RA ECs (online supplementary figure S4C-D). The restoration of SIRT1 expression in RA ECs using adenovirus transduction conducted to a significant reduction of their proliferation rate compared with mock-transduced cells (slope analysis with best-fit values: $0.29 \pm 0.01$ vs $0.26 \pm 0.01, \mathrm{p}=0.004$ ) 

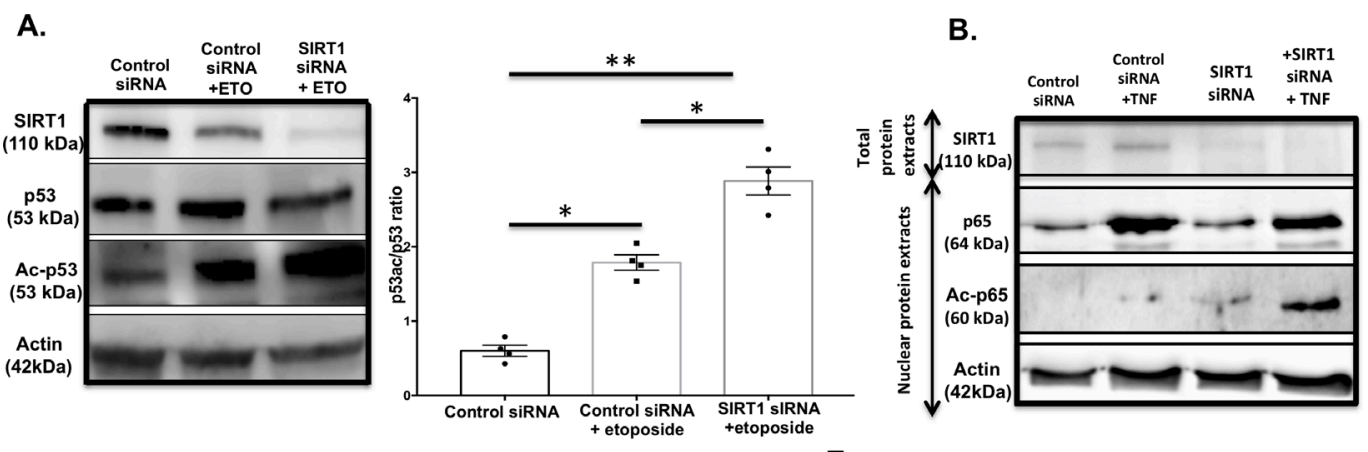

B.

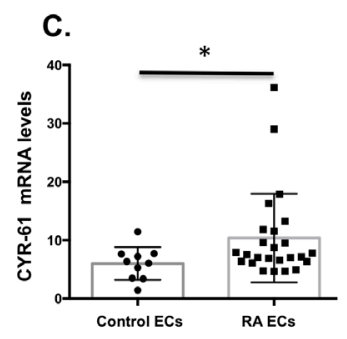

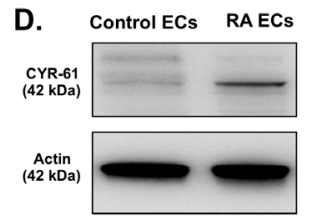

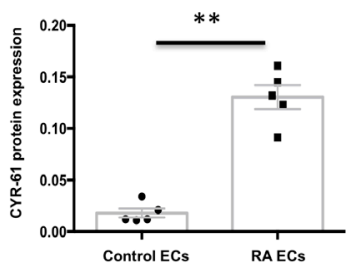

E. Control ECs

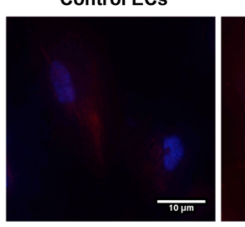

F.

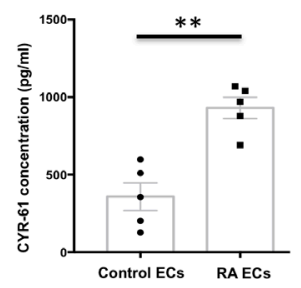

RA ECs

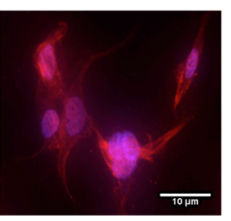

G.
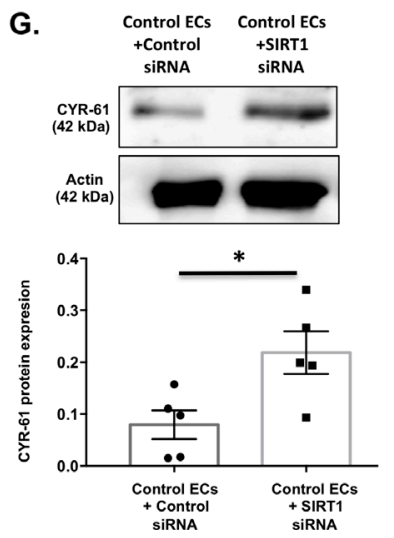

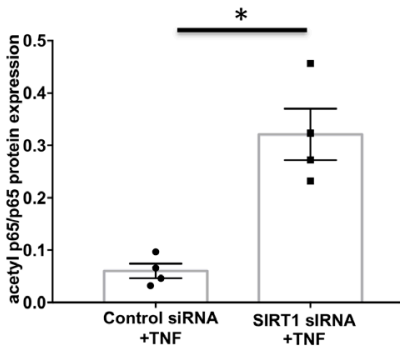

Figure 6 Mechanism of action of sirtuin-1 (SIRT1) in control endothelial cells (ECs). (A) Cell extracts from mock-transfected and SIRT1-transfected control ECs were immunoblotted for acetylated (Ac)-p53 and p53 after etoposide exposure ( $100 \mu \mathrm{M}$ for 24 hours). Y-axis represents the Ac-p53/p53 ratio after etoposide exposure $(100 \mu \mathrm{M}$ for 24 hours) in mock-transfected $(n=4)$ and SIRT1-transfected $(n=4)$ control ECs. (B) Cell extracts from mocktransfected and SIRT1-transfected control ECs were immunoblotted for Ac-p65 and p65 following tumour necrosis factor (TNF)- $\alpha$ stimulation (50 ng/ $\mathrm{mL}$ for 5 hours). Y-axis shows the Ac-p65/p65 ratio on TNF- $\alpha$ stimulation $(50 \mathrm{ng} / \mathrm{mL}$ for 5 hours) in mock-transfected ( $\mathrm{n}=4)$ and SIRT1-transfected $(n=4)$ control ECs. (C) Relative CYR61 mRNA levels quantified by qRT-PCR in RA ECs ( $n=25)$ and control ECs ( $n=9)$. (D) Cell extracts from cultured RA and control ECs were immunoblotted for CYR61. Y-axis shows the quantification of anti-CYR61 by western blot analysis. (E) Representative immunofluorescence staining for CYR61 in RA and control ECs (scale bar=10 $\mu \mathrm{m}$ ). Nuclei are stained with DAPI (blue). (F) Quantification of fluorescence intensity with ImageJ. (G) Cell extracts from mock-transfected and SIRT1-transfected control ECs were immunoblotted for CYR61. Y-axis shows the quantification of anti-CYR61 by western blot analysis. ECs from five independent patients with RA and five independent controls were used in all experiments, unless stated otherwise. All data are shown as the mean $\pm S E M .{ }^{*} P<0.05,{ }^{*} p<0.01$ determined by Student's $t$-test $(B, C$, $D, F, G$ ) or one-way analysis of variance with Tukey's post hoc test $(A)$ for experiments including more than two groups in one experiment. Data are representative of three independent experiments.

(figure 7A). Apoptosis of RA ECs was also significantly reduced on adenoviral SIRT1 overexpression, with a $40 \%$ decrease of the number of Annexin V+/PI- cells on exposure to etoposide $(\mathrm{p}=0.010)$ (figure $7 \mathrm{~B})$.

SIRT1 overexpression alleviated TNF- $\alpha$-induced activation of RA ECs: RA ECs transduced with SIRT1 adenovirus displayed a significant reduction of VEGF mRNA expression, VEGF release in culture supernatants $(44 \%, \mathrm{p}=0.011)$, adhesion molecule expression (figure 7C) and stress fibre formation (figure 7D). Adenoviral overexpression of SIRT1 in RA ECs also reversed the proangiogenic properties of RA ECs (figure 7E-F).

\section{In vivo modulation of SIRT in experimental arthritis}

SIRT1 conditional deletion in ECs increases angiogenesis and delays the resolution of experimental arthritis

Conditional endothelial deletion of SIRT1 did not have major effects on the initiation phase of arthritis or in the maximal intensity of arthritis observed at days 2 and 3, but led to longer persistence of arthritis (figure 8A-B). Indeed, mice with conditional invalidation of SIRT1 in ECs showed persistent signs of arthritis at day 7, whereas their wild-type littermates had an almost complete regression of arthritis (figure 8A-D). Histological analysis performed at day 7 showed more synovial alteration and pannus formation in the paw of mice invalidated for SIRT1 in ECs (figure 8E-F). These mice also displayed a striking increase of synovial vessel density (figure $8 \mathrm{G}, \mathrm{H}$ ). Conditional endothelial SIRT1 invalidation was also associated with a substantial increase of Ac-p53 (figure 8G-I), illustrating the loss of SIRT1 activity in the target tissue, and CYR61 expression in synovial vessels (figure 8G-J).

\section{Upregulation of SIRT1 alleviates experimental arthritis}

Resveratrol exerted anti-inflammatory effects during the initiation phase of arthritis and significantly reduced the maximal intensity of arthritis observed at day 2 (online supplementary figure S5A-D). Histological semi-quantitative score performed at day 6 was markedly reduced in the paw of resveratrol-treated mice (online supplementary figure S5E-F).

As expected, resveratrol led to the activation of SIRT1 activity, characterised by a striking reduction of p53 acetylation by $57 \%$ in the lesional synovial tissue (online supplementary figure S6A-C). 

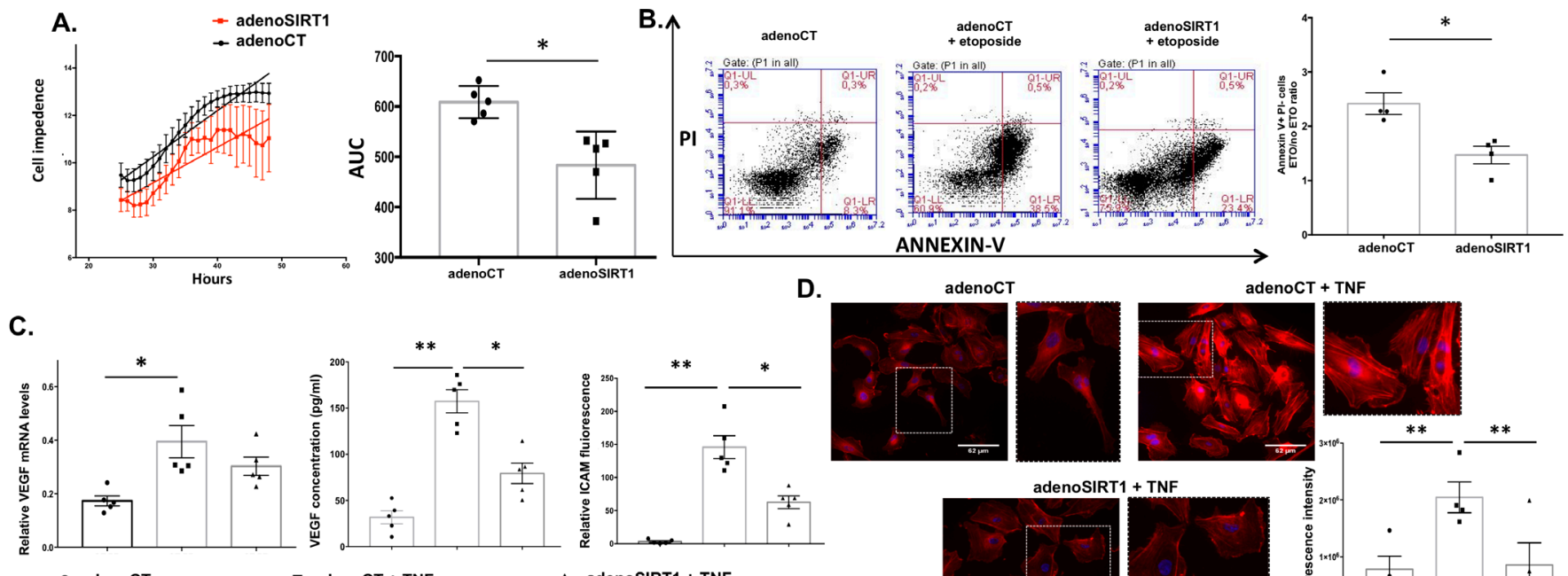

nocT

- adenoCT

- adenoCT + TNF

E.
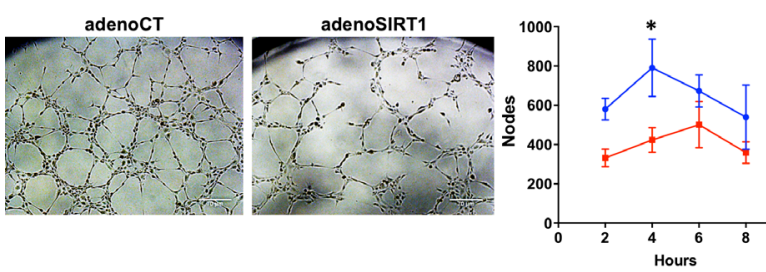

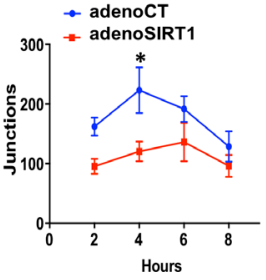

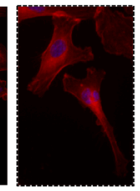
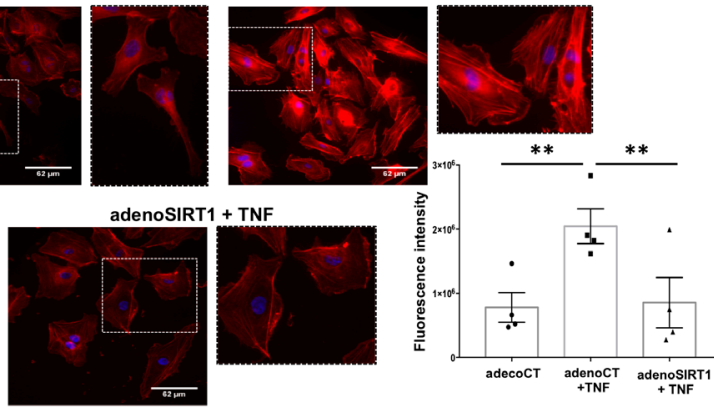

F.
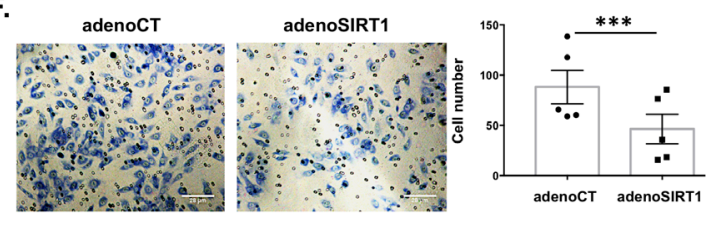

Figure 7 Effect of sirtuin-1 (SIRT1) activation on functional properties in rheumatoid arthritis (RA) endothelial cells (ECs). (A) Cell impedance measured by xCELLigence system in SIRT1-overexpressing RA ECs and mock-transduced RA ECs. Control and SIRT1 adenovirus (adenoCT and adenoSIRT1, respectively) has been added at H24. Y-axis shows the area under the curve of cell impedance. (B) Representative flow cytometry dot plots with double Annexin V-FITC/PI staining following etoposide-induced apoptosis (100 $\mu \mathrm{M}$ for 24 hours). Y-axis shows the $x$-fold change of Annexin V-FITC+/PI- cells after etoposide exposure $(100 \mu \mathrm{M}$ for 24 hours) in SIRT1-overexpressing RA ECs $(n=4)$ and mock-transduced RA ECs ( $n=4)$. (C) Relative vascular endothelial growth factor (VEGF) mRNA levels (qRT-PCR), VEGF concentration in culture cell supernatants (ELISA) and intercellular adhesion molecule (ICAM)-1 expression (flow cytometry) in SIRT1-overexpressing RA ECs and mock-transduced RA ECs following tumour necrosis factor (TNF)- $\alpha$ exposition ( $50 \mathrm{ng} / \mathrm{mL}$ for 5 hours). (D) Representative images of stress fibre formation on TNF- $\alpha$ stimulation ( $50 \mathrm{ng} / \mathrm{mL}$ for $5 \mathrm{hours}$ ) in SIRT1-overexpressing RA ECs $(n=4)$ and mock-transduced RA ECs $(n=4)$ (scale bar $=62 \mu \mathrm{m})$. Nuclei are stained with DAPI (blue). Y-axis shows the fluorescence intensity quantified by ImageJ. (E) Representative images of tube formation at 4 hours in SIRT1-overexpressing RA ECS and mocktransduced RA ECs (scale bar $=70 \mu \mathrm{m}$ ). Y-axis shows the analysis of node and junction numbers at 2, 4,6 and 8 hours. (F) Representative images of cell migration in modified Boyden chamber following vascular endothelial growth factor (VEGF) activation ( $50 \mathrm{ng} / \mathrm{mL}$ for 6 hours) in SIRT1-overexpressing RA ECs and mock-transduced RA ECs (scale bar $=28 \mu \mathrm{m}$ ). Y-axis shows the analysis of the number of migrated cells. ECs from five independent patients with RA and five independent controls were used in all experiments, unless stated otherwise. All data are shown as the mean \pm SEM. ${ }^{*} P<0.05$, ${ }^{* *} \mathrm{p}<0.01,{ }^{* *} \mathrm{p}<0.001$ determined by Student's t-test $(\mathrm{A}, \mathrm{B}, \mathrm{E}, \mathrm{F})$ or one-way analysis of variance with Tukey's post hoc test $(\mathrm{C}, \mathrm{D})$ for experiments including more than two groups in one experiment. Data are representative of two independent experiments.

\section{DISCUSSION}

Our results provide the experimental evidence of a major role of ECs derived from circulating progenitors in RA. Moreover, we identified in SIRT1 a relevant actor involved in all the main pathological features of those cells.

Although isolated from peripheral blood, and not directly from the synovium, ECs derived from circulating progenitors may be directly implicated in RA pathogenesis. Cells expressing progenitor markers have been detected in RA synovial tissue, ${ }^{21}$ supporting their homing in the pathological synovium. Moreover, early passage progenitor-derived RA ECs display in vitro a proliferative, activated and proangiogenic profile, possibly triggered by RA local and systemic inflammatory microenvironment. ${ }^{22}$ The number of circulating endothelial progenitor cell also correlate with RA disease activity indices. ${ }^{23}$

Gene expression analysis of RA ECs revealed a high number of differentially expressed genes involved in tumourigenesis. Moreover, SIRT1 was identified through a network constituted of highly differentially expressed genes implicated in cancer processes, possibly related to chronic exposition to metabolic stress signals, including hypoxia and inflammatory cytokines. ${ }^{24}$ SIRT1 is a NAD-dependent protein deacetylase that links transcriptional regulation to a variety of metabolic signals. ${ }^{25}$ Decreased endothelial SIRT1 expression is consistent with SIRT1 underexpression previously reported in RA fibroblast-like synoviocytes and peripheral blood mononuclear cells. ${ }^{24} 26$ Together with decreased SIRT1 expression, diminished lysyl deacetylase activity was detected in RA ECs. This was not related to decreased substrate availability, given the increased p53 and p65 acetylation in RA ECs, but rather to post-translational modifications. Indeed, miR-217 and miR-181a were able to specifically target SIRT1 in RA ECs. ${ }^{27}$ miR-181a is known to inhibit SIRT1 expression by directly binding to the 3 ' untranslated region of SIRT1 mRNA and miR-217 was shown to be important in senescence by inhibiting SIRT1, reducing nitric oxide availability and deacetylating Forkhead Box O1. ${ }^{13}$

SIRT1 silencing in control ECs reproduced the proliferative, pro-apoptotic, activated and proangiogenic profile of RA ECs (online supplementary figure S7), and these effects were reversed by adenoviral SIRT1 overexpression. The regulation of cell 
A.

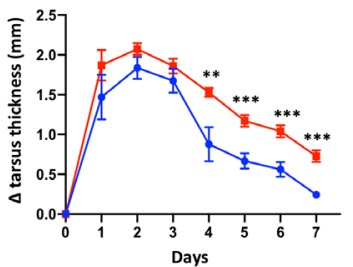

B.

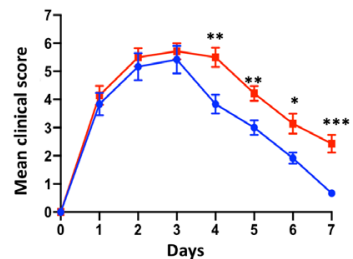

E.

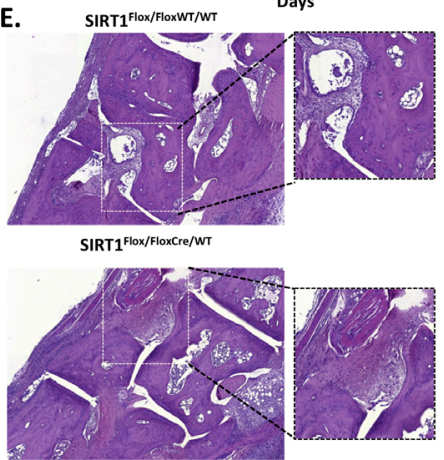

C.

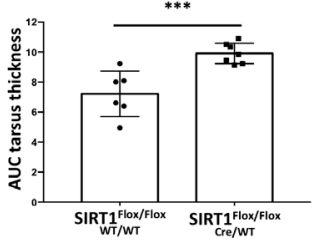

D.

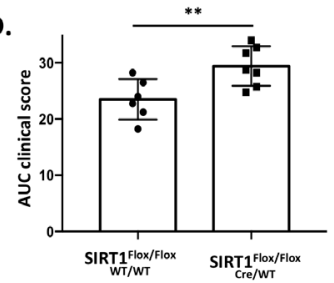

F.

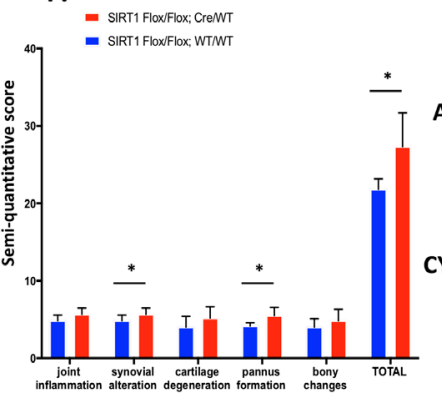

G.

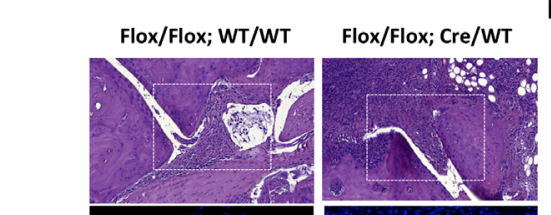

DAPI
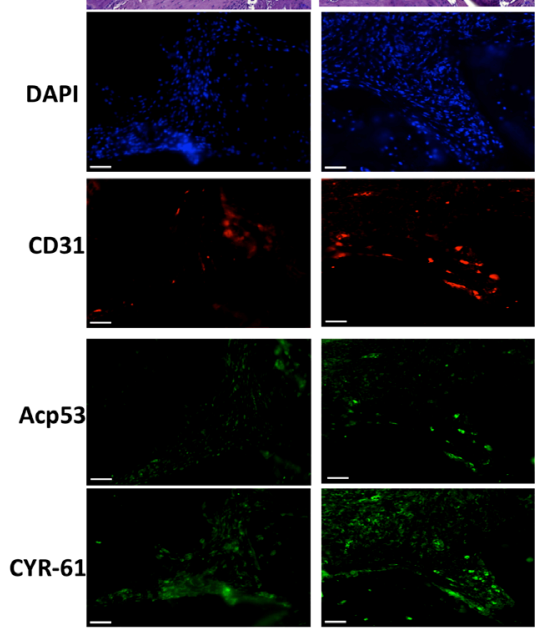

H.

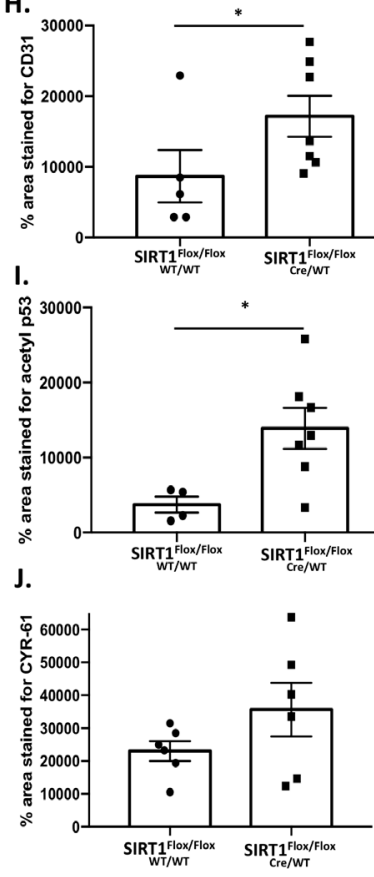

Figure 8 Effect of sirtuin-1 (SIRT1) endothelial invalidation on experimental arthritis. (A-D) Methyl-bovine serum albumin-(mBSA)-induced arthritis in littermates of SIRT1 Flox/Flox; WT/WT mice $(n=6)$ and SIRT1 Flox/Flox; Cre/WT mice $(n=7)$; Y-axis shows tarsus thickness (A) and clinical score (B), as well as the area under the curve (AUC) of tarsus thickness (C) and the clinical score (D). (E) Sections of ankle and tarsus joints from SIRT ${ }^{\text {Flox/Flox;WT/WT }}$ mice and SIRT1 $1^{\text {Flox/Flox; Cre/WT }}$ mice stained with H\&E at day 7 of arthritis (scale bar=100 $\mu \mathrm{m}$ ). (F) Histomorphometric analysis of the area of synovitis and bone

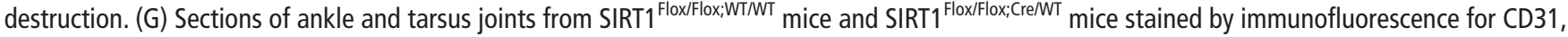
acetylated (Ac)-p53 and CYR61 (scale bar=50 $\mu \mathrm{m}$ ). Nuclei are stained with DAPI (blue). (H-J) Quantification of CD31 (H), Ac-p53 (I) and CYR61 (J) fluorescence intensity with ImageJ. All data are shown as the mean \pm SEM. ${ }^{*} \mathrm{P}<0.05,{ }^{* *} \mathrm{p}<0.01,{ }^{* *} \mathrm{p}<0.001$ determined by Student's t-test. Data are representative of a single (A-F) or two independent experiments ( $G-J)$.

proliferation is a key downstream effect of SIRT1, with various cell type-specific effects. ${ }^{28-31}$ Invalidation of SIRT1 was associated with increased EC apoptosis mediated by the upregulation of p53 acetylation, which is indispensable for p53 transcriptional activity. ${ }^{32}$ SIRT1 effects on apoptosis remain elusive, ${ }^{33} 34$ and discrepancies may be related to SIRT1 subcellular localisation. ${ }^{35} 36$

Endothelial SIRT1 invalidation markedly increased EC sensitisation to the proinflammatory cytokine TNF- $\alpha$, through the acetylation of the NF- $\kappa B$ family protein $\mathrm{p} 65$. NF- $\kappa \mathrm{B}$ is constitutively activated in RA and maintains a damaging phenotype of several cell types in RA. ${ }^{37}$ The transcriptional activity of p65 could be further regulated by phosphorylation and acetylation. Endothelial invalidation of SIRT1 did not inhibit the induction of p 65 by TNF- $\alpha$, but was associated with increased p65 acetylation, which is required for p65 full transcriptional activity, ${ }^{38}$ and led to amplification of TNF- $\alpha$-induced response and EC activation. Thus, SIRT1 may serve as a regulator of the NF- $\kappa B$ pathway in ECs, coordinating multiple downstream signals that may interact to reduce synovial inflammation.

Invalidation of SIRT1 in control ECs was also associated with increased proangiogenic properties and increased expression of the matricellular protein CYR61, which is essential for the control of angiogenesis. Moreover, CYR61 was upregulated in RA ECs and arthritic mice with conditional endothelial invalidation of SIRT1 displayed increased vessel density and higher CYR61 expression. In line with our results, inhibition of SIRT1/ FoxO3a signalling has been shown to be crucial to induce CYR61 in RA synovial fibroblasts, since forced SIRT1 expression in RA synovial fibroblasts led to decreased CYR61 levels. ${ }^{18}$ Interestingly, serum CYR61 levels were significantly increased in patients with RA and its concentrations were inversely correlated with RA disease activity and upregulated in those therapeutic responders. ${ }^{39}$

Recent evidence has suggested primary involvement of angiogenesis in the initiation of tissue inflammation prior to infiltration of inflammatory cells. Indeed, angiogenesis may precede leucocyte infiltration during inflammation in experimental models of inflammatory diseases. ${ }^{6}$ However, the effect of increased angiogenesis through conditional endothelial invalidation of SIRT1 did not modify the initial phase of arthritis, but resulted in a longer resolution phase of experimental arthritis. This is likely due to the features of mBSA model, in which the initial phase of arthritis is characterised by a potent inflammatory response even in control mice, which may have masked an early effect of SIRT1 invalidation. SIRT1 activation by Resveratrol reduced the maximal intensity of arthritis in the initial phase experimental arthritis, as previously described in complementary preclinical models, ${ }^{40-42}$ which may have direct therapeutic implications. Indeed, targeting angiogenesis, and especially SIRT1, might be used as a complementary therapeutic approach in RA.

\footnotetext{
Author affiliations

${ }^{1}$ INSERM U1016 and CNRS UMR8104, Institut Cochin, Paris, France

${ }^{2}$ Université Versailles St-Quentin, Signalisation et Physiopathologie Cardiovasculaire - UMR-S 1180, Univ Paris-Sud, INSERM, Université Paris-Saclay, Châtenay-Malabry, France

${ }^{3}$ UMR 1125 INSERM, Bobigny, France

${ }^{4}$ Sorbonne Paris Cité Université Paris 13, Bobigny, France
} 
${ }^{5}$ Service de Médecine Interne, Fondation Rothschild, Paris, France

6INSERM UMR-S 999, Hôpital Marie Lannelongue, Le Plessis-Robinson, France

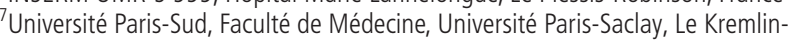
Bicêtre, France

${ }^{8}$ Service de Rhumatologie, GH Avicenne-Jean Verdier-René Muret, APHP, Bobigny, France

${ }^{9}$ Université de Paris, Université Paris Descartes, Paris, France

${ }^{10}$ Service de Rhumatologie, Hôpital Cochin, AP-HP.CUP, Paris, France

Acknowledgements Research Facilities of the Cochin Institute, INSERM U1016 and CNRS UMR8104, Paris, France: GENOM'IC : Genome and Sequencing (Franck Letourneur, Florent Dumont, Sébastien Jacques), CYBIO: Cytometry and Immunobiology (Muriel Andrieu), HistlM: Morphology and Histology (Maryline Favier) IMAGIC: Cellular Imaging, Confocal Microscopy (Pierre Bourdoncle). Julie Burlot, Valérie Domergue, AnimEx, UMS IPSIT, Faculté de Pharmacie, Châtenay-Malabry, France.Carole Nicco and Frederic Batteux, INSERM U1016 and CNRS UMR8104, Cochin Institute, Paris, France.

Contributors Study design: JA, YA, CL. Conduction of experiments: AL, SP, AC, CC, JPDS, RH, GC. Data analysis: AL, RH, GC, NB, LS, CL, YA, JA. Providing mice and samples: SD, SC-K. Writing/drafting and revisiting the manuscript: $A L, J A, L S, C L, Y A$ JA. Final approval of the manuscript: AL, SP, AC, CC, JPDS, RH, GC, SD, SC-K, NB, LS, $C L, Y A$, JA.

Funding This study was funded by Société Française de Rhumatologie, Arthritis R\&D, Bourse Passerelle (Pfizer).

Competing interests JA has received research funding for this study from Pfizer.

Patient and public involvement Patients and/or the public were not involved in the design, conduct, reporting or dissemination plans of this research.

Patient consent for publication Not required.

Provenance and peer review Not commissioned; externally peer reviewed.

Data availability statement All data obtained by microarray analysis have been deposited in a public, open access repository: GEO Omnibus site with the accession number GSE121894 (available at the following webpage: https://www.ncbi.nlm. nih.gov/geo/query/acc.cgi?acc=GSE121894). The authors declare that all other data supporting the findings of this study are included in the article or uploaded as supplementary information.

\section{ORCID ID}

Jérôme Avouac http://orcid.org/0000-0002-2463-218X

\section{REFERENCES}

1 Fries JF, Bloch DA. Mortality in rheumatoid arthritis patients. Ann Rheum Dis 1999;58:723-4

2 Leblond A, Allanore Y, Avouac J. Targeting synovial neoangiogenesis in rheumatoid arthritis. Autoimmun Rev 2017;16:594-601.

3 Elshabrawy HA, Chen Z, Volin MV, et al. The pathogenic role of angiogenesis in rheumatoid arthritis. Angiogenesis 2015;18:433-48

4 Szade A, Grochot-Przeczek A, Florczyk U, et al. Cellular and molecular mechanisms of inflammation-induced angiogenesis. IUBMB Life 2015;67:145-59.

5 Szekanecz Z, Koch AE. Mechanisms of disease: angiogenesis in inflammatory diseases. Nat Clin Pract Rheumatol 2007:3:635-43.

6 Riesner K, Shi Y, Jacobi A, et al. Initiation of acute graft-versus-host disease by angiogenesis. Blood 2017:129:2021-32.

7 Avouac J, Wipff J, Goldman 0, et al. Angiogenesis in systemic sclerosis: impaired expression of vascular endothelial growth factor receptor 1 in endothelial progenitor-derived cells under hypoxic conditions. Arthritis Rheum 2008;58:3550-61.

8 Avouac J, Guignabert C, Hoffmann-Vold AM, et al. Role of stromelysin 2 (matrix metalloproteinase 10) as a novel mediator of vascular remodeling underlying pulmonary hypertension associated with systemic sclerosis. Arthritis Rheumatol 2017;69:2209-21

9 Avouac J, Cagnard N, Distler JH, et al. Insights into the pathogenesis of systemic sclerosis based on the gene expression profile of progenitor-derived endothelial cells. Arthritis Rheum 2011:63:3552-62.

10 Kuwana M, Okazaki Y. Brief report: impaired in vivo neovascularization capacity of endothelial progenitor cells in patients with systemic sclerosis. Arthritis Rheumatol 2014;66:1300-5.

11 Arnett FC, Edworthy SM, Bloch DA, et al. The American rheumatism association 1987 revised criteria for the classification of rheumatoid arthritis. Arthritis Rheum 1988;31:315-24.

12 Aletaha D, Neogi T, Silman AJ, et al. Rheumatoid arthritis classification criteria: an American College of Rheumatology/European League against rheumatism collaborative initiative. Arthritis Rheum 2010;2010:2569-81.
13 Menghini R, Casagrande V, Cardellini M, et al. Microrna 217 modulates endothelial cell senescence via silent information regulator 1. Circulation 2009;120:1524-32.

14 Choi S-E, Kemper JK. Regulation of SIRT1 by microRNAs. Mol Cells 2013;36:385-92.

15 Cui C, Li Y, Liu Y. Down-Regulation of miR-377 suppresses high glucose and hypoxiainduced angiogenesis and inflammation in human retinal endothelial cells by direct up-regulation of target gene SIRT1. Hum Cell 2019;32:260-74.

16 Grandela C, Pera MF, Grimmond SM, et al. P53 is required for etoposide-induced apoptosis of human embryonic stem cells. Stem Cell Res 2007;1:116-28.

17 Yeung F, Hoberg JE, Ramsey CS, et al. Modulation of NF-kappaB-dependent transcription and cell survival by the SIRT1 deacetylase. Embo J 2004;23:2369-80.

18 Kok S-H, Lin L-D, Hou K-L, et al. Simvastatin inhibits cysteine-rich protein 61 expression in rheumatoid arthritis synovial fibroblasts through the regulation of sirtuin-1/FoxO3a signaling. Arthritis Rheum 2013:65:639-49.

19 Kwon E-J, Park E-J, Yu H, et al. Sirt-1 regulates TGF- $\beta$-induced dermal fibroblast migration via modulation of Cyr61 expression. Connect Tissue Res 2018;59:245-54.

20 Babic AM, Kireeva ML, Kolesnikova TV, et al. Cyr61, a product of a growth factorinducible immediate early gene, promotes angiogenesis and tumor growth. Proc Natl Acad Sci U S A 1998;95:6355-60.

21 Rüger B, Giurea A, Wanivenhaus AH, et al. Endothelial precursor cells in the synovial tissue of patients with rheumatoid arthritis and osteoarthritis. Arthritis Rheum 2004;50:2157-66.

22 Blandizzi C, Gionchetti P, Armuzzi A, et al. The role of tumour necrosis factor in the pathogenesis of immune-mediated diseases. Int J Immunopathol Pharmacol 2014;27:1-10

23 Jodon de Villeroché V, Avouac J, Ponceau A, et al. Enhanced late-outgrowth circulating endothelial progenitor cell levels in rheumatoid arthritis and correlation with disease activity. Arthritis Res Ther 2010;12:R27.

24 Li G, Xia Z, Liu Y, et al. Sirt1 inhibits rheumatoid arthritis fibroblast-like synoviocyte aggressiveness and inflammatory response via suppressing NF- $\kappa$ B pathway. Biosci Rep 2018;38

25 Vachharajani VT, Liu T, Wang $X$, et al. Sirtuins link inflammation and metabolism. J Immunol Res 2016;2016:1-10

26 Wendling D, Abbas W, Godfrin-Valnet M, et al. Dysregulated serum IL-23 and SIRT1 activity in peripheral blood mononuclear cells of patients with rheumatoid arthritis. PLoS One 2015:10:e0119981.

27 Revollo JR, Li X. The ways and means that fine tune SIRT1 activity. Trends Biochem SCi 2013:38:160-7.

28 Rathbone CR, Booth FW, Lees SJ. Sirt1 increases skeletal muscle precursor cell proliferation. Eur J Cell Biol 2009:88:35-44.

29 Kabra N, Li Z, Chen L, et al. Sirt1 is an inhibitor of proliferation and tumor formation in colon cancer. J Biol Chem 2009;284:18210-7.

30 Zu Y, Liu L, Lee MYK, et al. Sirt1 promotes proliferation and prevents senescence through targeting LKB1 in primary porcine aortic endothelial cells. Circ Res 2010:106:1384-93.

31 Cho I-R, Koh SS, Malilas W, et al. Sirt1 inhibits proliferation of pancreatic cancer cells expressing pancreatic adenocarcinoma up-regulated factor (PAUF), a novel oncogene, by suppression of $\beta$-catenin. Biochem Biophys Res Commun 2012;423:270-5.

32 Brooks CL, Gu W. The impact of acetylation and deacetylation on the p53 pathway. Protein Cell 2011;2:456-62.

33 Peng J-S, Chen S-Y, Wu C-L, et al. Amelioration of experimental autoimmune arthritis through targeting of synovial fibroblasts by intraarticular delivery of microRNAs 140$3 p$ and 140-5p. Arthritis Rheumatol 2016;68:370-81

34 Nakayama H, Yaguchi T, Yoshiya S, et al. Resveratrol induces apoptosis MH7A human rheumatoid arthritis synovial cells in a sirtuin 1-dependent manner. Rheumatol In 2012;32:151-7.

35 Jin Q, Yan T, Ge X, et al. Cytoplasm-Localized SIRT1 enhances apoptosis. J Cell Physiol 2007:213:88-97.

36 Tanno M, Sakamoto J, Miura T, et al. Nucleocytoplasmic shuttling of the NAD+dependent histone deacetylase SIRT1. J Biol Chem 2007:282:6823-32.

37 Sun C-L, Wei J, Bi L-Q. Rutin attenuates oxidative stress and proinflammatory cytokine level in adjuvant induced rheumatoid arthritis via inhibition of NF-кB. Pharmacology 2017:100:40-9.

38 Chen L-feng, Mu Y, Greene WC. Acetylation of RelA at discrete sites regulates distinct nuclear functions of NF-kappaB. Embo J 2002:21:6539-48.

39 Fan $Y$, Yang X, Zhao J, et al. Cysteine-Rich 61 (CYR61): a biomarker reflecting disease activity in rheumatoid arthritis. Arthritis Res Ther 2019:21:123.

40 Xuzhu G, Komai-Koma M, Leung BP, et al. Resveratrol modulates murine collagen-induced arthritis by inhibiting Th17 and B-cell function. Ann Rheum Dis 2012;71:129-35

41 Riveiro-Naveira RR, Valcárcel-Ares MN, Almonte-Becerril M, et al. Resveratrol lowers synovial hyperplasia, inflammatory markers and oxidative damage in an acute antigen-induced arthritis model. Rheumatology 2016;55:1889-900.

42 Khojah HM, Ahmed S, Abdel-Rahman MS, et al. Resveratrol as an effective adjuvant therapy in the management of rheumatoid arthritis: a clinical study. Clin Rheumato 2018:37:2035-42. 